\title{
Ácido Aconítico em Sementes de Espécies de Plantas Daninhas DE DIFERENTES LOCAIS ${ }^{1}$
}

\author{
Aconitic Acid on Seeds of Weed Species from Different Locations
}

VOLL, E. ${ }^{2}$, GAZZIERO, D.L.P. ${ }^{2}$ e ADEGAS, F.S. ${ }^{2}$

\begin{abstract}
RESUMO - Plantas em estado vegetativo, como trigo ou aveia usados nos sistemas de cultivo de soja, podem produzir e liberar substâncias alelopáticas pelas suas raízes, afetando espécies de plantas daninhas, somando-se aos efeitos produzidos pelas suas palhadas. Experimentos foram conduzidos em laboratório com o objetivo de determinar os efeitos do ácido aconítico sobre as espécies de plantas daninhas amendoim-bravo (Euphorbia heterophylla), corda-deviola (Ipomoea grandifolia), picão-preto (Bidens pilosa) e guanxuma (Sida rhombifolia), provenientes de diferentes locais do Estado do Paraná. Os ensaios constaram de tratamentos com e sem ácido aconítico $2,5 \mathrm{mM} \mathrm{L}^{-1}$. Corda-de-viola recebeu um pré-tratamento de escarificação com ácido sulfúrico. As sementes foram esterilizadas externamente com solução de hipoclorito de sódio a $2 \%$ durante dois minutos e enxaguadas. Em capela asséptica, em gerbox contendo meio de cultura de ágar, foram dispostas na superficie 50 sementes/ recipiente. Os experimentos foram conduzidos em câmara de germinação controlada. O delineamento utilizado foi o inteiramente casualizado, com quatro repetições. A origem das sementes teve influência nos resultados obtidos. A germinação das sementes foi afetada pelos efeitos do ácido aconítico na maioria dos locais. Ocorreu também a redução do crescimento das plântulas, sendo mais afetadas as raizes do que o caule, nas quatro espécies. O ácido aconítico apresenta efeitos alelopáticos sobre as sementes de diferentes espécies de plantas daninhas, variáveis com o local de origem, estimulando o crescimento de diferentes fungos endofiticos. Os efeitos do ácido aconítico podem traduzir-se na redução do período de sobrevivência dos bancos de sementes no solo.
\end{abstract}

Palavras-chave: alelopatia, fungos endofíticos, banco de sementes, dinâmica de populações.

\begin{abstract}
Plants in vegetative state, such as wheat or oat used in soybean crops, may produce and release allellopathic substances through their roots, affecting weed species, complemented by the effects produced by straw. Experiments were carried out under laboratory conditions to determine the effects of aconitic acid on weed species such as wild poinsettia (Euphorbia heterophylla), morningglory (Ipomoea grandifolia), prickly sida (Sida rhombifolia) and beggarticks (Bidens pilosa) originated from different locations in the state of Paraná. Experiments consisted of treatments with and without AA 2,5 $\mathrm{mM} \mathrm{L}^{-1}$. Morningglory received a pre-treatment with sulfuric acid. The weed species seeds were sterilized externally using sodium hypochloride solution at $2 \%$ during two minutes and then rinsed. In an aseptic hood, in a gerbox containing agar medium, 50 seeds/container were placed on the surface. Experiments were arranged in a completely randomized design, with four replications, placed in a germination chamber. Seed origin influenced the results obtained. In general, seed germination was affected by aconitic acid in most locations. Growth reduction of seedlings was observed, with the roots being more affected than the stems, in all the species. Aconitic acid presents allellopathic effects on different weed species, depending on their place of origin, stimulating the growth of several endophytic fungi. Aconitic acid effects may result in reduction of the survival period of the seedbanks in soil.
\end{abstract}

Keywords: allelopathy, endophytic fungi, seedbank, population dynamics.

1 Recebido para publicação em 8.2.2009 e na forma revisada em 12.3.2010.

2 Engo - -Agr ${ }^{0}$, Dr., Pesquisador em Plantas Daninhas da Embrapa Soja, Caixa Postal 231, 86001-970 Londrina-PR, $<$ voll@cnpso.embrapa.br>

Planta Daninha, Viçosa-MG, v. 28, n. 1, p. 13-22, 2010 


\section{INTRODUÇÃO}

Efeitos alelopáticos sobre espécies daninhas podem ser causados por plantas em estado vegetativo, ou pela decomposição de seus restos vegetais de palhadas. A permeabilidade do tegumento das sementes daninhas às substâncias exsudadas pelas suas raízes das plantas também permite que fungos endofiticos das sementes sejam estimulados a se desenvolver e a destruir aquelas ainda dormentes, reduzindo, assim, o banco de sementes.

Culturas como as de trigo (Thompson et al., 1997); pastagens de gramineas (Friebe et al., 1995), milho e sorgo; cevada e trigo (Rustamani et al., 1992); e, principalmente, cana-deaçúcar (Hanine et al., 1990) produzem e exsudam ácido aconítico através de suas raízes.

O ácido aconítico (AA), uma substância identificada em gramíneas, como em capimmarmelada (Brachiaria plantaginea), é responsável por efeitos alelopáticos e estímulo ao crescimento de fungos endofiticos de sementes de trapoeraba (Commelina benghalensis), conforme relatado por Voll et al. (2004). O AA resulta do metabolismo do açúcar na planta, apresentando a mesma fórmula molecular, porém a estrutural é modificada (Goodwin $\&$ Mercer, 1983). Outros relatos sobre o AA indicam importantes funções fisiológicas (Rustamani et al., 1992; Thompson et al., 1997; Watanabe et al., 1997). O ácido t-aconítico é comumente encontrado em clarificações de caldo de cana-de-açúcar (Hanine et al., 1990) ou na vinhaça. É um ácido orgânico de baixo peso molecular $(174,11 \mathrm{~g})$ e pode ser encontrado na solução do solo, juntamente com outros ácidos (Hees et al., 2000).

Diferenciais de comportamento de espécies de plantas daninhas, como de amendoimbravo, picão (Bidens pilosa) e trapoeraba (Voll et al., 1997, 2001), podem ser alterados nas suas capacidades de germinação e ter reduzida a sua sobrevivência no solo. Corda-deviola (Ipomoea aristolochiaefolia), referida como espécie de baixa capacidade germinativa, apresenta um tegumento bastante impermeável (Mikusinski, 1987), assim como de outras espécies do gênero Ipomoea, o que propicia a manutenção da viabilidade das sementes por longo tempo (Stoller \& Wax, 1973;
Chandler et al., 1977), podendo influenciar a absorção de substâncias alelopáticas. Sementes como as de balãozinho (Cardiospermum halicacabum) podem apresentar tegumento bastante impermeável, porém com a eliminação deste podem ocorrer germinação e emergência significativas (Johnston et al., 1979). Sementes que apresentam mucilagens, como as de amendoim-bravo, apresentam facilidade de germinação (Harper \& Benton, 1966), sendo influenciadas pela quantidade de chuva nos diferente locais. Diferentes condições de permeabilidade do tegumento de sementes à água podem interferir na absorção de substâncias alelopáticas. Outro fator a considerar é a produção de "sementes duras" (Stoller \& Wax, 1973), influenciadas pelas condições ambientais (Probert et al., 1985), incluindo-se os fatores genéticos (Chancellor, 1982).

O objetivo desta pesquisa foi determinar efeitos inibidores (alelopáticos) em sementes e estimulatórios de fungos endofiticos, produzidos pela ação do ácido aconítico, sobre algumas espécies de plantas daninhas, coletadas em diferentes locais do Estado do Paraná.

\section{MATERIAL E MÉTODOS}

Quatro experimentos foram conduzidos em laboratório, envolvendo as sementes das espécies de plantas daninhas amendoim-bravo (Euphorbia heterophylla), picão-preto (Bidens pilosa), corda-de-viola (Ipomoea grandifolia) e guanxuma (Sida rhombifolia), coletadas em diferentes locais do Estado do Paraná, no período de fevereiro a março de 2006 e 2007, e conservadas em câmara seca $\left(10^{\circ} \mathrm{C}\right.$ e UR do ar a $40 \%$ ), sendo testadas cerca de quatro meses mais tarde.

Ágar bacteriológico a $12 \mathrm{~g} \mathrm{~L}^{-1}$, introduzido em água destilada, foi autoclavado por 15 minutos a $120{ }^{\circ} \mathrm{C}$. Depois disso, o meio de ágar foi resfriado $\left(40-45^{\circ} \mathrm{C}\right)$ e adicionada a respectiva dose de $2,5 \mathrm{mM} \mathrm{L}^{-1}$ de AA puro para análise, no estado ainda líquido, cujo peso molecular é de $174,11 \mathrm{~g}$. Uma quantidade de $100 \mathrm{~mL}$ de cada tratamento foi introduzida em recipientes de plástico com tampa e deixada a resfriar para adquirir consistência sólida. Um tratamento testemunha, sem AA, foi preparado. Corda-de-viola recebeu um tratamento 
adicional de escarificação das sementes com ácido sulfúrico concentrado, durante cinco minutos, e enxaguada. As sementes foram, então, esterilizadas externamente com solução de hipoclorito de sódio a $2 \%$ durante dois minutos e enxaguadas por igual período. A seguir, em capela asséptica e com auxílio de uma pinça, foram dispostas na superficie do meio 50 sementes/recipiente, usando quatro repetições. O experimento foi colocado em câmara de germinação, com ciclos de luz e de temperatura de $14 / 10$ horas e $30^{\circ} \mathrm{C} / 20^{\circ} \mathrm{C}$, respectivamente.

O vigor das sementes foi avaliado após um periodo de 10-12 dias, por meio do percentual de germinação, quando esta cessou, e do crescimento, medindo-se o comprimento do caule e da raiz. Após um período de 5-7 dias, foi feita a identificação e a contagem dos diferentes tipos de fungos que contaminavam as sementes. Quanto ao amendoim-bravo, foram feitas análises químicas das sementes para alguns locais de coleta, a fim de identificar possiveis correlações de comportamento.

O delineamento usado foi o inteiramente casualizado, com arranjo fatorial e quatro repetições. Foi feita a análise da variância e o F-teste. A comparação entre as médias foi feita pelo teste de Tukey a $5 \%$.

\section{RESULTADOS E DISCUSSÃO}

\section{Efeitos do ácido aconitico (AA) em sementes de amendoim-bravo}

Os locais do Estado do Paraná foram ordenados de modo decrescente de germinação para o tratamento sem aplicação do AA. A taxa de germinação máxima e mínima de amendoimbravo foi de 95,0 e $16,0 \%$, respectivamente, indicando ocorrer alta capacidade de germinação e considerável amplitude no ano entre os locais (Tabela 1). O AA nem sempre manifestou seus efeitos inibitórios sobre a germinação das sementes provenientes dos diferentes locais, por meio de redução significativa. Niveis de redução da germinação, não significativos, podem ser constatados tanto nos percentuais altos de germinação das sementes sem AA quanto nos mais baixos. O AA reduziu a germinação em 13 locais, o comprimento do caule em 22 locais e a raiz do amendoim-bravo em todos os 24 locais. As reduções médias decorrentes dos efeitos alelopáticos foram de $16,2 \%$ na germinação, $40,3 \%$ no comprimento do caule e $84,4 \%$ no comprimento de raízes.

O estímulo de AA sobre o desenvolvimento de fungos endofiticos das sementes de amendoim-bravo foi avaliado e feita uma tentativa de identificação deles (Tabela 2). Dois tipos de fungo foram predominantes: Aspergillus sp. e Fusarium sp. Considerando-se as médias, apenas Aspergillus foi estimulado, o que foi possibilitado pela sua maior infestação, e, na ausência de AA, os locais não diferiram entre si. Fusarium apresentou variações com e sem AA nos diferentes locais e poucas vezes com diferenças significativas, em vista da menor infestação. O somatório de fungos assemelhouse no comportamento ao de Aspergillus. A presença do fungo Bipolaris foi observada no local Céu Azul, e a de Rhyzopus, em São Miguel, onde foi observado o estímulo significativo do AA. Prete et al. (1984) detectaram, em amostras de sementes de plantas daninhas de 15 espécies, a presença de esporos de fungos de diversos gêneros, como de Cladosporium, Alternaria, Penicilium, Aspergillus, Phoma, Rhizopus, Fusarium e outros. Os microrganismos endofiticos de sementes, como fungos, são considerados patógenos latentes (Azevedo $\&$ Melo, 1998). Os endófitos podem produzir toxinas e destruir as sementes. Efeitos alelopáticos e estímulos no desenvolvimento de fungos endofíticos foram observados em sementes de trapoeraba ( $C$. benghalensis) (Voll et al., 2004), em amendoim-bravo (E. heterophylla) e em corda-de-viola (I. grandifolia) (Voll et al., 2005). A predominância de espécies de fungos tende a variar com as espécies de plantas daninhas. A presença de AA em plantas de capim-marmelada (B. plantaginea) representou 95\% das substâncias alelopáticas (Voll et al., 2004). Na média geral, o AA estimulou o surgimento de fungos de 11,1 para $36,6 \%$, numa proporção de $1: 3,3$.

Uma análise química das sementes de amendoim-bravo, compreendendo a escolha de alguns locais e separação em classes de germinação, não mostrou relação com as características de germinação ou a presença de fungos (Tabela 3).

Planta Daninha, Viçosa-MG, v. 28, n. 1, p. 13-22, 2010 
Tabela 1 - Efeitos do ácido aconítico (AA) na germinação e no comprimento do caule e da raiz de amendoim-bravo, aos 12 dias, em diferentes locais do Estado do Paraná, em 2006

\begin{tabular}{|c|c|c|c|c|c|c|}
\hline \multirow{2}{*}{ Locais (24) } & \multicolumn{2}{|c|}{ Germinação (\%) } & \multicolumn{2}{|c|}{ Caule $(\mathrm{mm})$} & \multicolumn{2}{|c|}{ Raiz (mm) } \\
\hline & Sem AA & Com AA & Sem AA & Com AA & Sem AA & Com AA \\
\hline Toledo & $95,0 \mathrm{aA}$ & $94,0 \mathrm{aA}^{1 / \prime}$ & $62,9 \operatorname{ch} A$ & $28,3 \mathrm{dfB}$ & $47,7 \mathrm{bdA}$ & $5,2 \mathrm{acB}$ \\
\hline Santa Terezinha I & $89,5 \mathrm{abA}$ & $64,0 \mathrm{beB}$ & $64,3 \mathrm{chA}$ & $35,1 \mathrm{afB}$ & $33,5 \mathrm{ehA}$ & $4,1 \mathrm{acB}$ \\
\hline Formosa do Oeste & $88,0 \mathrm{abA}$ & $66,0 \mathrm{beB}$ & $66,6 \mathrm{bgA}$ & $33,8 \mathrm{bfB}$ & $49,0 \mathrm{bcA}$ & $9,2 \mathrm{acB}$ \\
\hline Ubiratã & $86,5 \mathrm{acA}$ & $42,0 \mathrm{ehB}$ & $81,0 \mathrm{abA}$ & $40,0 \mathrm{adB}$ & $39,0 \mathrm{cfA}$ & $3,0 \mathrm{acB}$ \\
\hline Matelândia & $85,5 \mathrm{acA}$ & $46,0 \mathrm{ehB}$ & 61,0diA & $51,2 \mathrm{aB}$ & 31,1 eiA & $4,7 \mathrm{acB}$ \\
\hline Campo Mourão & $83,5 \mathrm{acA}$ & $84,5 \mathrm{acA}$ & $57,4 \mathrm{ejA}$ & $32,7 \mathrm{cfB}$ & 35,1 egA & $3,3 \mathrm{acB}$ \\
\hline Santa Terezinha II & $83,0 \mathrm{acA}$ & $67,0 \mathrm{beB}$ & $60,1 \mathrm{diA}$ & $35,4 \mathrm{afB}$ & $41,1 \mathrm{ceA}$ & $2,0 \mathrm{bcB}$ \\
\hline Assis & $82,5 \mathrm{acA}$ & $88,5 \mathrm{abA}$ & $78,7 \mathrm{acA}$ & $33,1 \mathrm{bfB}$ & $53,7 \mathrm{bA}$ & $5,8 \mathrm{acB}$ \\
\hline Medianeira & $77,9 \mathrm{dfA}$ & $53,5 \mathrm{adB}$ & $31,9 \mathrm{~mA}$ & 42,7afA & 29,9eiA & $9,0 \mathrm{cB}$ \\
\hline Bragantina & $75,0 \mathrm{adA}$ & $74,5 \mathrm{adA}$ & $45,8 \mathrm{imA}$ & $20,9 \mathrm{fB}$ & $37,0 \operatorname{dgA}$ & $7,3 \mathrm{acB}$ \\
\hline Bandeirante do Oeste & $74,5 \mathrm{adA}$ & $57,5 \mathrm{dfB}$ & $72,4 \mathrm{bfA}$ & $37,0 \mathrm{afB}$ & $55,6 \mathrm{bA}$ & $5,2 \mathrm{acB}$ \\
\hline Quinta do Sol & 73,0adA & $80,0 \mathrm{adA}$ & $73,7 \mathrm{beA}$ & $39,3 \mathrm{adB}$ & $50,2 \mathrm{bcA}$ & $1,5 \mathrm{cB}$ \\
\hline Janiópolis I & $70,5 \mathrm{aeA}$ & 81,5 beA & $91,9 \mathrm{aA}$ & $34,8 \mathrm{bfB}$ & $72,0 \mathrm{aA}$ & $7,8 \mathrm{acB}$ \\
\hline São Pedro & $69,5 \mathrm{beA}$ & $48,5 \mathrm{egB}$ & $73,4 \mathrm{bfA}$ & $47,7 \mathrm{acB}$ & $37,1 \mathrm{dg} \mathrm{A}$ & $6,2 \mathrm{acB}$ \\
\hline Campo Mourão & 69,0 beA & $34,0 \mathrm{fiB}$ & $78,2 \mathrm{acA}$ & $42,0 \mathrm{adB}$ & $36,9 \mathrm{dgA}$ & $2,8 \mathrm{bcB}$ \\
\hline Tupãssi & 66,0 beA & $60,0 \mathrm{ceA}$ & $48,3 \mathrm{hlA}$ & $37,1 \mathrm{afB}$ & $27,9 \mathrm{fiA}$ & $4,5 \mathrm{acB}$ \\
\hline Cafelândia & $62,0 \mathrm{cfA}$ & $42,5 \mathrm{ehB}$ & $57,3 \mathrm{fjA}$ & $38,4 \mathrm{aeB}$ & $34,5 \operatorname{eg} \mathrm{A}$ & $5,2 \mathrm{acB}$ \\
\hline Mamborê & $51,5 \mathrm{dfA}$ & 30,0 giB & $65, \mathrm{bgA}$ & $33,3 \mathrm{bfB}$ & 27,1 giA & $12,1 \mathrm{acB}$ \\
\hline São Miguel & $45,5 \operatorname{egA}$ & $24,0 \mathrm{giB}$ & $61,0 \mathrm{~mA}$ & $22,1 \mathrm{efB}$ & $31,1 \mathrm{hiA}$ & $3,2 \mathrm{acB}$ \\
\hline Vera Cruz do Oeste & $39,0 \mathrm{fhA}$ & $23,0 \mathrm{hiB}$ & $53,0 \mathrm{gkA}$ & $34,6 \mathrm{bfB}$ & $28,7 \mathrm{fiA}$ & $13,5 \mathrm{abB}$ \\
\hline Céu Azul & $38,0 \mathrm{fhA}$ & $48,0 \mathrm{ehA}$ & $42,0 \mathrm{jmA}$ & $49,3 \mathrm{abA}$ & $21,4 \mathrm{iA}$ & $6,0 \mathrm{acB}$ \\
\hline Tupãssi & $22,0 \mathrm{ghA}$ & $25,5 \mathrm{giA}$ & $75,4 \mathrm{bdA}$ & $46,8 \mathrm{acB}$ & $28,6 \mathrm{fiA}$ & $14,7 \mathrm{aB}$ \\
\hline Jataizinho & $20,0 \mathrm{hA}$ & $23,5 \mathrm{giA}$ & $36,6 \operatorname{lm} \mathrm{A}$ & $28,5 \mathrm{dfA}$ & 34,9 egA & $7,1 \mathrm{acB}$ \\
\hline Peabiru & $16,0 \mathrm{hA}$ & $9,0 \mathrm{iA}$ & $38,6 \mathrm{kmA}$ & $27,0 \mathrm{dfB}$ & $32,4 \mathrm{eiA}$ & $6,4 a c B$ \\
\hline Médias & $64,1 \mathrm{~A}$ & $53,7 \mathrm{~B}$ & $60,3 \mathrm{~A}$ & $36,0 \mathrm{~B}$ & $37,8 \mathrm{~A}$ & $5,9 \mathrm{~B}$ \\
\hline $\mathrm{CV}(\%)$ & \multicolumn{2}{|c|}{16,1} & \multicolumn{2}{|c|}{12,9} & \multicolumn{2}{|c|}{20,3} \\
\hline
\end{tabular}

1/ Médias seguidas pelas mesmas letras, minúsculas nas colunas e maiúsculas nas linhas, não diferem entre si pelo teste de Tukey a $5 \%$.

\section{Efeitos de ácido aconitico (AA) em sementes de picão-preto}

As taxas de germinação e os comprimentos do caule e da raiz de picão-preto apresentaram diferenças significativas entre os diferentes locais de origem das sementes sem $\mathrm{AA}$, as quais desapareceram com AA, que reduziu significativamente as médias dos parâmetros analisados (Tabela 4). Os efeitos inibitórios de AA foram significativos em relação à germinação na maioria das vezes. A variabilidade dos fatores analisados foi reduzida entre os locais com a aplicação do AA.

A germinação de picão entre os locais variou numa amplitude de 32,0 a 47,8\%, na ausência de AA. O AA reduziu a germinação de picão em cerca de $26,8 \%$, em média, e o crescimento do caule e da raiz em 82,8 e $76,9 \%$, respectivamente.

A ocorrência de fungos endofiticos nas sementes de picão indicou a predominância de três espécies, citando-se Fusarium > Bipolaris $>$ e Aspergillus (Tabela 5). Aspergillus não apresentou diferenças entre os locais de origem das sementes ou maior presença do fungo em função da aplicação do AA. A infestação de Bipolaris também não apresentou diferenças entre os locais de origem das sementes, ocorrendo estímulo na maioria com a aplicação do AA, verificando-se aumento significativo na média geral. Para Fusarium, não houve diferenças entre os locais e nas suas médias, com ou sem a aplicação de AA, havendo aumento da infestação do fungo com a aplicação do AA em parte dos locais. No 
Tabela 2 - Ocorrência e intensidade de fungos endofíticos em sementes de amendoim-bravo, com e sem ácido aconítico (AA), em diferentes locais do Estado do Paraná, em 2006

\begin{tabular}{|c|c|c|c|c|c|c|}
\hline \multirow{2}{*}{ Locais (24) } & \multicolumn{2}{|c|}{ Aspergillus sp. } & \multicolumn{2}{|c|}{ Fusarium sp. } & \multicolumn{2}{|c|}{ spp. $(\%)$} \\
\hline & Sem AA & Com AA & Sem AA & Com AA & Sem AA & Com AA \\
\hline Toledo & $0,3 \mathrm{aA}$ & $2,0 \mathrm{gA}^{\frac{1}{1}}$ & $0,3 \mathrm{dA}$ & $0,0 \mathrm{dA}$ & $1,0 \mathrm{bcA}$ & $4,0 \mathrm{iA}$ \\
\hline Santa Terezinha I & $0,5 \mathrm{aB}$ & $9,0 \mathrm{fgA}$ & $0,5 \mathrm{~dB}$ & $8,5 \mathrm{abA}$ & $2,5 \mathrm{bcB}$ & $35,0 \mathrm{ehA}$ \\
\hline Formosa do Oeste & $0,8 \mathrm{aA}$ & $2,3 \mathrm{gA}$ & $4,5 \mathrm{bdB}$ & $9,0 \mathrm{aA}$ & $10,5 \mathrm{bcB}$ & $27,5 \mathrm{fiA}$ \\
\hline Ubiratã & $0,0 \mathrm{aB}$ & $32,0 \mathrm{aA}$ & $0,8 \mathrm{dA}$ & $0,5 \mathrm{dA}$ & $3,0 \mathrm{bcB}$ & $65,5 \mathrm{abA}$ \\
\hline Matelândia & $2,0 \mathrm{aB}$ & $24,8 \mathrm{acA}$ & $2,0 \mathrm{cdA}$ & $3,0 \mathrm{bdA}$ & $8,0 \mathrm{bcB}$ & $58,0 \mathrm{aeA}$ \\
\hline Campo Mourão & $2,8 \mathrm{aA}$ & $4,5 f g A$ & $1,0 \mathrm{dA}$ & $2,8 \mathrm{bdA}$ & $7,5 \mathrm{bcA}$ & 15,0giA \\
\hline Santa Terezinha II & $0,0 \mathrm{aB}$ & $5,8 \mathrm{fg} \mathrm{A}$ & $0,0 \mathrm{dA}$ & $1,0 \mathrm{cdA}$ & $0,0 \mathrm{cA}$ & $24,5 \mathrm{fiA}$ \\
\hline Assis & $0,8 \mathrm{aA}$ & $1,8 \mathrm{gA}$ & $2,5 \mathrm{cdA}$ & $1,5 \mathrm{cdA}$ & $7,5 \mathrm{bcA}$ & $7,5 \mathrm{iA}$ \\
\hline Medianeira & $0,0 \mathrm{aA}$ & $2,0 \mathrm{gA}$ & $3,5 \mathrm{bdA}$ & $4,0 \mathrm{bdA}$ & $7,0 \mathrm{bcA}$ & 17,0giA \\
\hline Bragantina & $1,8 \mathrm{aA}$ & $6,3 f g A$ & $0,5 \mathrm{dA}$ & $0,3 \mathrm{dA}$ & $4,5 \mathrm{bcA}$ & $15,5 \mathrm{giA}$ \\
\hline Bandeirante do Oeste & $8,3 \mathrm{aB}$ & $19,8 \mathrm{bdA}$ & $0,3 \mathrm{dA}$ & $0,0 \mathrm{dA}$ & $17,5 \mathrm{bcB}$ & $41,0 \mathrm{cfA}$ \\
\hline Quinta do Sol & $6,3 \mathrm{aA}$ & 9,5egA & $0,3 \mathrm{dA}$ & $0,0 \mathrm{dA}$ & $13,0 \mathrm{bcA}$ & 19,0 fiA \\
\hline Janiópolis & $0,0 \mathrm{aA}$ & $4,3 f g \mathrm{~A}$ & $1,0 \mathrm{dA}$ & $1,8 \mathrm{bdA}$ & $8,0 \mathrm{bcA}$ & 13,5hiA \\
\hline São Pedro & $2,3 \mathrm{aB}$ & $29,5 \mathrm{aA}$ & $1,5 \mathrm{cdA}$ & $0,8 \mathrm{cdA}$ & $7,5 \mathrm{bcB}$ & $60,5 \mathrm{adA}$ \\
\hline Campo Mourão & $1,8 \mathrm{aB}$ & $29,3 \mathrm{abA}$ & $0,5 \mathrm{dA}$ & $1,5 \mathrm{cdA}$ & $4,5 \mathrm{bcB}$ & $62,0 \mathrm{acA}$ \\
\hline Tupãssi & $0,0 \mathrm{aA}$ & 10,0 egA & $4,5 \mathrm{bdA}$ & $3,0 \mathrm{bdA}$ & $9,0 \mathrm{bcB}$ & 27,0 fiA \\
\hline Cafelândia & $0,5 \mathrm{aB}$ & $23,5 \mathrm{acA}$ & $4,3 \mathrm{bdA}$ & $4,0 \mathrm{bdA}$ & $9,5 \mathrm{bcB}$ & $55,0 \mathrm{aeA}$ \\
\hline Mamborê & $1,5 \mathrm{aB}$ & $19,0 \mathrm{ce} A$ & $8,3 \mathrm{bcA}$ & $7,5 \mathrm{acA}$ & $20,0 \mathrm{bcB}$ & $62,0 \mathrm{acA}$ \\
\hline São Miguel & $2,0 \mathrm{aA}$ & $5,8 \mathrm{fgA}$ & $4,0 \mathrm{bdA}$ & $4,0 \mathrm{bdA}$ & $24,5 \mathrm{abB}$ & $68,0 \mathrm{aA}$ \\
\hline Vera Cruz do Oeste & $0,5 \mathrm{aB}$ & $11,0 \operatorname{dg} \mathrm{A}$ & $4,8 \mathrm{bdA}$ & $6,3 \mathrm{adA}$ & $10,5 \mathrm{bcB}$ & $34,5 \mathrm{ehA}$ \\
\hline Céu Azul & $2,8 \mathrm{aB}$ & $19, \mathrm{bdA}$ & $6,0 \mathrm{bdA}$ & $1,0 \mathrm{cdB}$ & $19,0 \mathrm{bcB}$ & $42,0 \mathrm{bfA}$ \\
\hline Tupãssi & $2,8 \mathrm{aB}$ & $23,3 \mathrm{acA}$ & $20,8 \mathrm{aA}$ & $7,5 \mathrm{acB}$ & $48,0 \mathrm{aB}$ & $61,5 \mathrm{dA}$ \\
\hline Jataizinho & $0,0 \mathrm{aB}$ & $13,5 \mathrm{dfA}$ & $10,0 \mathrm{bA}$ & $5,3 \mathrm{bdB}$ & $20,0 \mathrm{bcB}$ & $38,0 \operatorname{dg} \mathrm{A}$ \\
\hline Peabiru & $0,0 \mathrm{aB}$ & $8,0 f g A$ & $2,5 \mathrm{cdA}$ & $4,3 \mathrm{bdA}$ & $5,0 \mathrm{bcB}$ & $24,5 \mathrm{fiA}$ \\
\hline Médias & $1,6 \mathrm{~B}$ & $13,2 \mathrm{~A}$ & $3,5 \mathrm{~A}$ & $3,2 \mathrm{~A}$ & $11,1 \mathrm{~B}$ & $36,6 \mathrm{~A}$ \\
\hline $\mathrm{CV}(\%)$ & \multicolumn{2}{|c|}{49,4} & \multicolumn{2}{|c|}{78,1} & \multicolumn{2}{|c|}{38,1} \\
\hline
\end{tabular}

1/ Médias seguidas pelas mesmas letras, minúsculas nas colunas e maiúsculas nas linhas, não diferem entre si pelo teste de Tukey a $5 \%$.

somatório das espécies de fungos, ocorreu maior variabilidade da presença entre as origens das sementes, nem sempre aumentada com a aplicação do AA. Na média, houve aumento da infestação de 6,2 para $11,0 \%$.

O picão-preto apresenta absorção rápida de água num período mínimo de tempo, em horas, resultando em emergência de até 87,5 (Adegas et al., 2003), o que pode facilitar a absorção de AA. Por sua vez, uma embebição prolongada de um dia pode resultar numa germinação prejudicada (Reddy \& Singh, 1992).

\section{Efeitos do ácido aconítico (AA) em sementes de corda-de-viola}

As taxas de germinação de corda-de-viola, em locais sem AA, foram semelhantes em seis dos sete locais (Tabela 6), variando entre 7,5 e $28,0 \%$. Os comprimentos do caule e da raiz também apresentaram variações entre locais.

$\mathrm{Na}$ média dos tratamentos com AA, a germinação não foi afetada por ele; no entanto, foi aumentada pela escarificação das sementes (AS+AA), devido aos efeitos significativos da interação desse tratamento com os locais, em S. Antônio do Paraíso e Assaí. Por sua vez, os comprimentos do caule e da raiz foram significativamente reduzidos na presença do AA, não sendo alterados pelos efeitos do ácido sulfúrico aplicado anteriormente.

Corda-de-viola $($ Ipomoea aristolochiaefolia $=$ grandifolia), referida como espécie de baixa capacidade germinativa, apresenta um tegumento bastante impermeável (Mikusinski, 1987), 
Tabela 3 - Análise química de sementes de amendoim-bravo, coletadas em 14 locais do Estado do Paraná

\begin{tabular}{|c|c|c|c|c|c|c|c|c|c|c|c|}
\hline \multirow{2}{*}{$\begin{array}{c}\text { Locais } \\
\text { (Sem AA) }\end{array}$} & $\mathrm{N}$ & $P$ & $\mathrm{~K}$ & $\mathrm{Ca}$ & $\mathrm{Mg}$ & $\mathrm{S}$ & $\mathrm{Zn}$ & $\mathrm{Mn}$ & $\mathrm{Fe}$ & $\mathrm{Cu}$ & B \\
\hline & \multicolumn{6}{|c|}{$\left(\mathrm{g} \mathrm{kg}^{-1}\right)$} & \multicolumn{5}{|c|}{$\left(\mathrm{mg} \mathrm{kg}^{-1}\right)$} \\
\hline \multicolumn{12}{|c|}{ Germinação: $0-50 \%$} \\
\hline Peabiru & 55,9 & 4,7 & 11,0 & 9,2 & 3,6 & 1,5 & 42,7 & 29,1 & 40,2 & 12,8 & 15,0 \\
\hline Tupãssi & 61,0 & 6,0 & 10,6 & 11,9 & 3,5 & 1,4 & 52,1 & 50,4 & 99,9 & 14,7 & 15,1 \\
\hline Jataizinho & 63,1 & 6,9 & 12,2 & 10,9 & 4,3 & 1,8 & 42,2 & 27,1 & 52,0 & 23,2 & 16,6 \\
\hline Céu Azul & 46,9 & 5,7 & 12,9 & 9,7 & 4,1 & 1,7 & 50,7 & 45,1 & 62,0 & 17,1 & 17,6 \\
\hline \multicolumn{12}{|c|}{ Germinação: $51-80 \%$} \\
\hline São Pedro & 65,6 & 7,4 & 11,4 & 10,6 & 4,2 & 1,6 & 62,4 & 39,4 & 48,4 & 15,9 & 2,5 \\
\hline Medianeira & 43,0 & 7,3 & 10,6 & 9,1 & 4,1 & 1,7 & 71,3 & 44,2 & 41,6 & 12,4 & 2,3 \\
\hline Bragantina & 66,8 & 8,6 & 16,0 & 12,7 & 5,7 & 2,1 & 109,7 & 60,3 & 86,8 & 16,5 & 7,1 \\
\hline Campo Mourão & 44,8 & 8,5 & 12,5 & 11,0 & 4,8 & 1,5 & 52,0 & 26,3 & 82,0 & 14,3 & 2,0 \\
\hline Cafelândia & 64,6 & 8,4 & 11,6 & 11,5 & 5,1 & 1,5 & 51,2 & 33,6 & 64,2 & 19,7 & 10,5 \\
\hline \multicolumn{12}{|c|}{ Germinação: 81-100\% } \\
\hline Ubiratã & 42,5 & 5,5 & 8,6 & 14,9 & 3,7 & 1,5 & 41,1 & 57,4 & 51,8 & 15,6 & 7,4 \\
\hline Matelândia & 64,5 & 6,8 & 12,0 & 9,8 & 4,1 & 1,5 & 49,0 & 57,8 & 50,8 & 13,9 & 2,4 \\
\hline Campo Mourão & 61,1 & 7,7 & 11,7 & 11,0 & 4,3 & 1,5 & 58,7 & 65,9 & 54,1 & 12,8 & 9,4 \\
\hline Janiópolis & 47,8 & 6,2 & 12,7 & 10,3 & 4,1 & 1,5 & 61,7 & 44,7 & 65,1 & 16,8 & 19,2 \\
\hline Toledo & 63,3 & 7,3 & 10,7 & 12,0 & 5,0 & 1,6 & 51,2 & 86,0 & 64,9 & 16,1 & 7,7 \\
\hline Máximo & 66,8 & 8,6 & 16,0 & 14,9 & 5,7 & 2,1 & 109,7 & 86,0 & 99,9 & 23,2 & 19,2 \\
\hline Mínimo & 42,5 & 4,7 & 8,6 & 9,1 & 3,5 & 1,4 & 41,1 & 26,3 & 40,2 & 12,4 & 2,0 \\
\hline
\end{tabular}

assim como de outras espécies do gênero Ipomoea, o que propicia a manutenção da viabilidade das sementes por longo tempo (Stoller \& Wax, 1973; Chandler et al., 1977), podendo influenciar a absorção de substâncias alelopáticas. Sementes como as de balãozinho (Cardiospermum halicacabum) podem apresentar tegumento bastante impermeável, porém com a sua eliminação pode ocorrer significativa germinação e emergência (Johnston et al., 1979).

A presença de Fusarium nas sementes de corda-de-viola, no tratamento "Sem AA", foi semelhante em seis dos sete locais (Tabela 7), não combinando com os niveis de germinação. A infestação variou entre 1,5 e 29,0\%, enquanto o somatório, com a inclusão de Bipolaris e de outras espécies (não citadas), variou entre 2,0 e 37,5\%, destacando-se em um dos locais. A presença do fungo Bipolaris foi baixa, com diferenças não significativas entre locais.

Nas médias dos tratamentos com AA (Tabela 7), não houve efeito significativo da aplicação desse ácido no percentual de Fusarium presente nas sementes de corda-de-viola, bem como para o somatório das espécies de fungos.
No entanto, o aumento foi significativo quando o ácido sulfúrico foi aplicado previamente na escarificação da espécie, aumentando também no seu somatório das espécies de fungos.

Para efeito dos tratamentos nos diversos locais (Tabela 7), observa-se que não houve estímulos significativos da aplicação de AA sobre os fungos Fusarium, Bipolaris ou no somatório das demais espécies identificadas, ocorrendo apenas com a escarificação prévia das sementes para Fusarium, em Santa Mariana (73,5\%) e Uraí (59,5\%). Contudo, no somatório das diversas espécies de fungos, a escarificação das sementes com ácido sulfúrico resultou em aumento significativo das infestações, ocorrendo em maior número de locais, citando-se Santa Mariana $(78,0 \%)$, Santo Antônio do Paraíso (51,5\%), Jataí (73,0\%) e Uraí $(73,0 \%)$.

Dez espécies de fungos foram identificadas na superficie das sementes: Fusarium, Bipolaris, Aspergillus, Rhizopus, Penicillium, Verticillium, Macrophomina, Tricoderma, Alternaria e Cladospermum, com predominância das duas primeiras. Muitas dessas espécies de fungos foram também identificadas por 
Prete et al. (1984) em amostras de sementes de plantas daninhas coletadas na região de Piracicaba.

\section{Efeitos de ácido aconítico (AA) em sementes de guanxuma}

Diferenças significativas de germinação de guanxuma ocorreram entre sementes coletadas em 11 locais, variando de 4,0 a $61,0 \%$, na ausência do ácido aconítico (Tabela 8). Também ocorreram variações significativas entre os locais para os comprimentos do caule e da raiz. A aplicação de AA não mostrou efeitos alelopáticos significativos na germinação de sementes coletadas em nove dos 11 locais. A germinação foi reduzida em $12,4 \%$, na média. No entanto, os comprimentos

Tabela 4 - Efeitos do ácido aconítico (AA) sobre a germinação e o crescimento do caule e da raiz de picão-preto, em diferentes locais do Estado do Paraná, em 2007

\begin{tabular}{|c|c|c|c|c|c|c|}
\hline \multirow{2}{*}{ Locais (12) } & \multicolumn{2}{|c|}{ Germinação (\%) } & \multicolumn{2}{|c|}{ Caule $(\mathrm{cm})$} & \multicolumn{2}{|c|}{ Raiz (cm) } \\
\hline & Sem AA & Com AA & Sem AA & Com AA & Sem AA & Com AA \\
\hline Campo Mourão & $38,8 \mathrm{adA}$ & $19,3 \mathrm{aB}^{1 /}$ & $2,09 \mathrm{abA}$ & $0,51 \mathrm{aB}$ & $1,83 \mathrm{abA}$ & $0,56 \mathrm{aB}$ \\
\hline IV Centenário & $41,5 \operatorname{adA}$ & $33,5 \mathrm{aB}$ & $3,19 \mathrm{ce} A$ & $0,51 \mathrm{aB}$ & $3,24 \mathrm{efA}$ & $0,71 \mathrm{aB}$ \\
\hline São Pedro & $32,0 \mathrm{aA}$ & $25,0 \mathrm{aA}$ & $2,62 \mathrm{acA}$ & $0,61 \mathrm{aB}$ & $2,19 \mathrm{acA}$ & $0,58 \mathrm{aB}$ \\
\hline São Miguel do Iguaçu & $35,0 \mathrm{acA}$ & $25,8 \mathrm{aB}$ & $2,41 \mathrm{acA}$ & $0,44 \mathrm{aB}$ & $2,76 \mathrm{ce} A$ & $0,63 \mathrm{aB}$ \\
\hline Jesuítas & $38,0 \mathrm{adA}$ & $25,8 \mathrm{aB}$ & $1,93 \mathrm{aA}$ & $0,41 \mathrm{aB}$ & $1,74 \mathrm{a} A$ & $0,67 \mathrm{aB}$ \\
\hline Formosa do Oeste & $38,8 \mathrm{adA}$ & $31,0 \mathrm{aB}$ & $3,18 \mathrm{ceA}$ & $0,36 \mathrm{aB}$ & $3,24 \mathrm{efA}$ & $0,48 \mathrm{aB}$ \\
\hline Nova Santa Bárbara & $33,8 \mathrm{abA}$ & $31,5 \mathrm{aA}$ & $2,68 \mathrm{adA}$ & $0,65 \mathrm{aB}$ & $2,15 \mathrm{acA}$ & $0,57 \mathrm{aB}$ \\
\hline Uraí & $45,0 \mathrm{bdA}$ & $34,3 \mathrm{aB}$ & $3,50 \mathrm{de} A$ & $0,61 \mathrm{aB}$ & $3,85 \mathrm{f} \mathrm{A}$ & $0,64 \mathrm{aB}$ \\
\hline São Judas Tadeu & $46,0 \mathrm{bdA}$ & $33,5 \mathrm{aB}$ & $3,76 \mathrm{eA}$ & $0,56 \mathrm{aB}$ & $2,83 \mathrm{ceA}$ & $0,58 \mathrm{aB}$ \\
\hline São Sebastião Amor & $44,8 \mathrm{bdA}$ & $32,0 \mathrm{aB}$ & $2,88 \mathrm{bdA}$ & $0,53 \mathrm{aB}$ & $2,96 \mathrm{deA}$ & $0,52 \mathrm{aB}$ \\
\hline Santa Cecília Pavão & $47,8 \mathrm{cA}$ & $37,5 \mathrm{aB}$ & $3,75 \mathrm{eA}$ & $0,53 \mathrm{aB}$ & $2,38 \mathrm{adA}$ & $0,51 \mathrm{aB}$ \\
\hline Cornélio Procópio & $47,3 \mathrm{cdA}$ & $28,5 \mathrm{aB}$ & $3,22 \mathrm{ce} A$ & $0,54 \mathrm{aB}$ & $2,46 \mathrm{cdA}$ & $0,55 \mathrm{aB}$ \\
\hline Médias & $40,7 \mathrm{~A}$ & $29,8 \mathrm{~B}$ & $2,9 \mathrm{~A}$ & $0,5 \mathrm{~B}$ & $2,6 \mathrm{~A}$ & $0,6 \mathrm{~B}$ \\
\hline $\mathrm{CV}(\%)$ & \multicolumn{2}{|c|}{14,6} & \multicolumn{2}{|c|}{19,1} & \multicolumn{2}{|c|}{19,2} \\
\hline
\end{tabular}

1/ Médias seguidas pelas mesmas letras, minúsculas nas colunas e maiúsculas nas linhas, não diferem entre si pelo teste de Tukey a 5\%.

Tabela 5 - Efeitos do ácido aconítico (AA) sobre a ocorrência de fungos endofíticos em sementes de picão-preto, em diferentes locais do Estado do Paraná, em 2007

\begin{tabular}{|c|c|c|c|c|c|c|c|c|}
\hline \multirow{3}{*}{ Locais (12) } & \multicolumn{2}{|c|}{ Aspergillus (\%) } & \multicolumn{2}{|c|}{ Bipolaris $(\%)$} & \multicolumn{2}{|c|}{ Fusarium $(\%)$} & \multicolumn{2}{|c|}{ spp. $(\%)$} \\
\hline & \multicolumn{8}{|c|}{ Ácido aconítico } \\
\hline & Sem & Com & Sem & Com & Sem & Com & Sem & Com \\
\hline Campo Mourão & 0,3 & 1,5 & $1,8 \mathrm{aB}^{\underline{1}}$ & $15,0 \mathrm{aA}$ & $2,8 \mathrm{aA}$ & $3,0 \mathrm{aA}$ & $4,8 \mathrm{acB}$ & $20,5 \mathrm{cA}$ \\
\hline IV Centenário & 0,5 & 0,8 & $2,5 \mathrm{aA}$ & $5,5 \mathrm{bA}$ & $4,5 \mathrm{aA}$ & $3,5 \mathrm{aA}$ & $7,8 \mathrm{acA}$ & $10,0 \mathrm{abA}$ \\
\hline São Pedro & 2,0 & 0,5 & $5,8 \mathrm{aA}$ & $4,5 \mathrm{bA}$ & $2,3 \mathrm{aA}$ & $2,8 \mathrm{aA}$ & $11,3 \mathrm{bcA}$ & $7,8 \mathrm{abA}$ \\
\hline São Miguel Iguaçu & 0,5 & 0,0 & $6,5 \mathrm{aB}$ & $7,8 \mathrm{abA}$ & $6,3 \mathrm{aB}$ & $2,3 \mathrm{aA}$ & $13,5 \mathrm{cA}$ & $10,5 \mathrm{acA}$ \\
\hline Jesuítas & 0,5 & 0,8 & $2,0 \mathrm{aB}$ & $6,8 \mathrm{bA}$ & $7,3 \mathrm{aB}$ & $4,0 \mathrm{aA}$ & $10,0 \mathrm{acA}$ & $11,5 \mathrm{acA}$ \\
\hline Formosa do Oeste & 1,0 & 0,3 & $1,5 \mathrm{aB}$ & $7,3 \mathrm{bA}$ & $4,0 \mathrm{aA}$ & $2,8 \mathrm{aA}$ & $7,0 \mathrm{acA}$ & $10,8 \mathrm{acA}$ \\
\hline Nova Santa Bárbara & 0,8 & 0,3 & $0,3 \mathrm{aA}$ & $4,5 \mathrm{bA}$ & $2,8 \mathrm{aA}$ & $2,8 \mathrm{aA}$ & $4,3 \mathrm{acA}$ & $7,5 \mathrm{abA}$ \\
\hline Uraí & 0,3 & 0,5 & $1,5 \mathrm{aB}$ & $6,5 \mathrm{bA}$ & $2,5 \mathrm{aA}$ & $4,5 \mathrm{aA}$ & $4,5 \mathrm{acB}$ & $11,5 \mathrm{acA}$ \\
\hline São Judas Tadeu & 0,0 & 0,0 & $0,0 \mathrm{aA}$ & $2,5 \mathrm{bA}$ & $0,3 \mathrm{aA}$ & $1,3 \mathrm{aA}$ & $0,3 \mathrm{aA}$ & $3,8 \mathrm{aA}$ \\
\hline São Sebastião Amor & 0,3 & 1,3 & $0,5 \mathrm{aB}$ & $5,8 \mathrm{bA}$ & $1,8 \mathrm{aB}$ & $5,0 \mathrm{aA}$ & $2,5 \mathrm{abB}$ & $14,3 \mathrm{bcA}$ \\
\hline Santa Cecília Pavão & 0,5 & 0,0 & $0,8 \mathrm{aA}$ & $3,5 \mathrm{bA}$ & $2,0 \mathrm{aA}$ & $4,0 \mathrm{aA}$ & $3,5 \mathrm{acA}$ & 9,3abA \\
\hline Cornélio Procópio & 0,5 & 0,0 & $0,0 \mathrm{aB}$ & $8,8 \mathrm{abA}$ & $3,3 \mathrm{aB}$ & $0,8 \mathrm{aA}$ & $4,3 \mathrm{acB}$ & $14,8 \mathrm{bcA}$ \\
\hline Médias & 0,6 & 0,5 & $1,9 \mathrm{~B}$ & $6,5 \mathrm{~A}$ & $3,3 \mathrm{~A}$ & $3,1 \mathrm{~A}$ & $6,2 \mathrm{~B}$ & $11,0 \mathrm{~A}$ \\
\hline $\mathrm{CV}(\%)$ & \multicolumn{2}{|c|}{ - } & \multicolumn{2}{|c|}{55,1} & \multicolumn{2}{|c|}{73,3} & \multicolumn{2}{|c|}{49,1} \\
\hline
\end{tabular}

${ }^{1 /}$ Médias seguidas pelas mesmas letras, minúsculas nas colunas e maiúsculas nas linhas, não diferem entre si pelo teste de Tukey a 5\%. 
Tabela 6 - Efeitos dos ácidos sulfúrico (AS) e aconítico (AA) sobre a germinação e o crescimento do caule e da raiz de corda-de-viola, em diferentes locais do Estado do Paraná, em 2007

\begin{tabular}{|c|c|c|c|c|}
\hline Variáveis & Tratamento & $\begin{array}{c}\text { Germinação } \\
(\%)\end{array}$ & $\begin{array}{c}\text { Caule } \\
(\mathrm{cm})\end{array}$ & $\begin{array}{l}\text { Raiz } \\
(\mathrm{cm})\end{array}$ \\
\hline \multicolumn{5}{|l|}{ Locais (7): - Sem AA } \\
\hline Santa Mariana & & $7,5 \mathrm{~b}^{-1}$ & $2,9 \mathrm{bc}$ & $3,2 \mathrm{ab}$ \\
\hline S. Antônio do Paraíso & & $18,0 \mathrm{ab}$ & $4,6 \mathrm{ab}$ & $4,8 \mathrm{a}$ \\
\hline Assaí & & $28,0 \mathrm{a}$ & $5,6 \mathrm{a}$ & $4,6 \mathrm{a}$ \\
\hline Nova Fátima & & $15,5 \mathrm{ab}$ & 3,8 abc & $3,2 \mathrm{ab}$ \\
\hline Jataí & & $12,5 \mathrm{ab}$ & $2,3 \mathrm{c}$ & $1,9 \mathrm{~b}$ \\
\hline Uraí & & $14,5 \mathrm{ab}$ & $3,3 \mathrm{bc}$ & $3,9 \mathrm{a}$ \\
\hline Cornélio Procópio & & $15,5 \mathrm{ab}$ & 3,8 abc & $2,1 \mathrm{~b}$ \\
\hline \multirow{3}{*}{ Tratamentos com AA } & Sem AA & $15,9 \mathrm{~b}$ & $3,7 \mathrm{a}$ & $3,4 \mathrm{a}$ \\
\hline & Com AA & $17,3 \mathrm{~b}$ & $1,0 \mathrm{~b}$ & $0,2 \mathrm{~b}$ \\
\hline & $\mathrm{AS}+\mathrm{AA}$ & $26,6 \mathrm{a}$ & $1,3 \mathrm{~b}$ & $0,2 \mathrm{~b}$ \\
\hline \multicolumn{5}{|l|}{ Tratamentos/Locais } \\
\hline \multirow{3}{*}{ Santa Mariana } & Sem AA & $7,5 \mathrm{a}$ & $2,9 \mathrm{a}$ & $3,2 \mathrm{a}$ \\
\hline & Com AA & $5,0 \mathrm{a}$ & $1,1 \mathrm{~b}$ & $0,2 \mathrm{~b}$ \\
\hline & $\mathrm{AS}+\mathrm{AA}$ & $16,0 \mathrm{a}$ & $2,1 \mathrm{ab}$ & $0,3 \mathrm{~b}$ \\
\hline \multirow{3}{*}{ S. Antônio do Paraíso } & Sem AA & $18,0 \mathrm{~b}$ & $4,6 \mathrm{a}$ & $4,8 \mathrm{a}$ \\
\hline & Com AA & $18,5 \mathrm{~b}$ & $1,0 \mathrm{~b}$ & $0,3 \mathrm{~b}$ \\
\hline & $\mathrm{AS}+\mathrm{AA}$ & $31,0 \mathrm{a}$ & $1,5 \mathrm{~b}$ & $0,2 \mathrm{~b}$ \\
\hline \multirow{3}{*}{ Assaí } & Sem AA & $28,0 \mathrm{~b}$ & $5,6 \mathrm{a}$ & $4,6 \mathrm{a}$ \\
\hline & Com AA & $21,5 \mathrm{~b}$ & $1,1 \mathrm{~b}$ & $0,3 \mathrm{~b}$ \\
\hline & AS+AA & $70,0 \mathrm{a}$ & $1,1 \mathrm{~b}$ & $0,2 \mathrm{~b}$ \\
\hline \multirow{3}{*}{ Nova Fátima } & Sem AA & $15,5 \mathrm{a}$ & $3,8 \mathrm{a}$ & $3,2 \mathrm{a}$ \\
\hline & Com AA & $19,5 \mathrm{a}$ & $1,0 \mathrm{~b}$ & $0,4 \mathrm{~b}$ \\
\hline & $\mathrm{AS}+\mathrm{AA}$ & $23,0 \mathrm{a}$ & $1,5 \mathrm{~b}$ & $0,2 \mathrm{~b}$ \\
\hline \multirow{3}{*}{ Jataí } & Sem AA & $12,5 \mathrm{a}$ & $2,2 \mathrm{a}$ & $1,9 \mathrm{a}$ \\
\hline & Com AA & $18,5 \mathrm{a}$ & $0,6 \mathrm{~b}$ & $0,2 \mathrm{~b}$ \\
\hline & $\mathrm{AS}+\mathrm{AA}$ & $12,0 \mathrm{a}$ & $0,8 \mathrm{~b}$ & $0,2 \mathrm{~b}$ \\
\hline \multirow{3}{*}{ Uraí } & Sem AA & $14,5 \mathrm{a}$ & $3,2 \mathrm{a}$ & $3,9 \mathrm{a}$ \\
\hline & Com AA & $18,0 \mathrm{a}$ & $1,4 \mathrm{~b}$ & $0,2 \mathrm{~b}$ \\
\hline & $\mathrm{AS}+\mathrm{AA}$ & $21,0 \mathrm{a}$ & $1,3 \mathrm{~b}$ & $0,2 \mathrm{~b}$ \\
\hline \multirow{3}{*}{ Cornélio Procópio } & Sem AA & $15,5 \mathrm{a}$ & $3,8 \mathrm{a}$ & $2,1 \mathrm{a}$ \\
\hline & Com AA & $20,0 \mathrm{a}$ & $0,7 \mathrm{~b}$ & $0,2 \mathrm{~b}$ \\
\hline & $\mathrm{AS}+\mathrm{AA}$ & $13,0 \mathrm{a}$ & $0,9 \mathrm{~b}$ & $0,2 \mathrm{~b}$ \\
\hline $\mathrm{CV}(\%)$ & & 37,7 & 43,7 & 59,9 \\
\hline
\end{tabular}

${ }^{1 /}$ Médias seguidas das mesmas letras nas colunas não diferem entre si pelo teste de Tukey a $5 \%$.
Tabela 7 - Efeitos do ácido sulfúrico (AS) e aconítico (AA) sobre a ocorrência de fungos endofíticos em sementes de corda-de-viola, em diferentes locais do Estado do Paraná, em 2007

\begin{tabular}{|c|c|c|c|c|}
\hline Variáveis & Tratamento & $\begin{array}{c}\text { Fusarium } \\
(\%)\end{array}$ & \begin{tabular}{|c} 
Bipolaris \\
$(\mathrm{cm})$
\end{tabular} & $\begin{array}{l}\text { spp. } \\
(\%)\end{array}$ \\
\hline \multicolumn{5}{|l|}{ Locais (7): - Sem AA } \\
\hline Santa Mariana & & $1,5 \mathrm{a}^{1 /}$ & $0,0 \mathrm{a}$ & $2,0 \mathrm{a}$ \\
\hline S. Antônio do Paraíso & & $5,0 \mathrm{ab}$ & $1,0 \mathrm{a}$ & $9,5 \mathrm{a}$ \\
\hline Assaí & & $9,0 \mathrm{ab}$ & $0,5 \mathrm{a}$ & $9,5 \mathrm{a}$ \\
\hline Nova Fátima & & $13,0 \mathrm{ab}$ & $3,5 \mathrm{a}$ & $19,0 \mathrm{ab}$ \\
\hline Jataí & & $16,5 \mathrm{ab}$ & $6,0 \mathrm{a}$ & $22,5 \mathrm{ab}$ \\
\hline Uraí & & $21,0 \mathrm{ab}$ & $0,0 \mathrm{a}$ & $25,5 \mathrm{ab}$ \\
\hline Cornélio Procópio & & $29,0 \mathrm{~b}$ & $8,5 \mathrm{a}$ & $37,5 \mathrm{~b}$ \\
\hline \multirow{3}{*}{ Tratamentos com AA } & Sem AA & $13,6 a^{\frac{1}{1 /}}$ & $2,8 \mathrm{a}$ & $17,9 \mathrm{a}$ \\
\hline & Com AA & $11,9 \mathrm{a}$ & $5,2 \mathrm{a}$ & $18,6 \mathrm{a}$ \\
\hline & $\mathrm{AS}+\mathrm{AA}$ & $29,8 \mathrm{~b}$ & $2,4 \mathrm{a}$ & $55,1 \mathrm{~b}$ \\
\hline \multicolumn{5}{|l|}{ Tratamentos/Locais } \\
\hline \multirow{3}{*}{ Santa Mariana } & Sem AA & $1,5 \mathrm{a}^{-1}$ & $0,0 \mathrm{a}$ & $2,0 \mathrm{a}$ \\
\hline & Com AA & $3,5 \mathrm{a}$ & $1,5 \mathrm{a}$ & $5,5 \mathrm{a}$ \\
\hline & $\mathrm{AS}+\mathrm{AA}$ & $73,5 \mathrm{~b}$ & $0,0 \mathrm{a}$ & $78,0 \mathrm{~b}$ \\
\hline \multirow{3}{*}{ S. Antônio do Paraíso } & Sem AA & $5,0 \mathrm{a}$ & $1,0 \mathrm{a}$ & $9,5 \mathrm{a}$ \\
\hline & Com AA & $7,5 \mathrm{a}$ & $2,0 \mathrm{a}$ & $9,5 \mathrm{a}$ \\
\hline & $\mathrm{AS}+\mathrm{AA}$ & $5,0 \mathrm{a}$ & $7,5 \mathrm{a}$ & $51,5 \mathrm{~b}$ \\
\hline \multirow{3}{*}{ Assaí } & Sem AA & $9,0 \mathrm{a}$ & $0,5 \mathrm{a}$ & $9,5 \mathrm{a}$ \\
\hline & Com AA & $9,5 \mathrm{a}$ & $1,5 \mathrm{a}$ & $11,5 \mathrm{a}$ \\
\hline & $\mathrm{AS}+\mathrm{AA}$ & $15,5 \mathrm{a}$ & $1,0 \mathrm{a}$ & $20,5 \mathrm{a}$ \\
\hline \multirow{3}{*}{ Nova Fátima } & Sem AA & $13,0 \mathrm{a}$ & $3,5 \mathrm{a}$ & $19,0 \mathrm{a}$ \\
\hline & Com AA & $16,5 \mathrm{a}$ & $5,0 \mathrm{a}$ & $24,0 \mathrm{a}$ \\
\hline & $\mathrm{AS}+\mathrm{AA}$ & $7,5 \mathrm{a}$ & $0,0 \mathrm{a}$ & $33,0 \mathrm{a}$ \\
\hline \multirow{3}{*}{ Jataí } & Sem AA & $16,5 \mathrm{a}$ & $6,0 \mathrm{a}$ & $22,5 \mathrm{a}$ \\
\hline & Com AA & $10,5 \mathrm{a}$ & $8,0 \mathrm{a}$ & $20,5 \mathrm{a}$ \\
\hline & $\mathrm{AS}+\mathrm{AA}$ & 18,5 a & $8,0 \mathrm{a}$ & $73,0 \mathrm{~b}$ \\
\hline \multirow{3}{*}{ Uraí } & Sem AA & $21,0 \mathrm{a}$ & $0,0 \mathrm{a}$ & $25,5 \mathrm{a}$ \\
\hline & Com AA & $19,0 \mathrm{a}$ & $2,5 \mathrm{a}$ & $22,5 \mathrm{a}$ \\
\hline & $\mathrm{AS}+\mathrm{AA}$ & $59,5 \mathrm{~b}$ & $0,0 \mathrm{a}$ & $73,0 \mathrm{~b}$ \\
\hline \multirow{3}{*}{ Cornélio Procópio } & Sem AA & 29,0 a & $8,5 \mathrm{a}$ & $37,5 \mathrm{a}$ \\
\hline & Com AA & $17,0 \mathrm{a}$ & $6,0 \mathrm{a}$ & $36,5 \mathrm{a}$ \\
\hline & $\mathrm{AS}+\mathrm{AA}$ & 29,0 a & $0,0 \mathrm{a}$ & 56,5 a \\
\hline $\mathrm{CV}(\%)$ & & 68,0 & 156,4 & 46,2 \\
\hline
\end{tabular}

${ }^{1 /}$ Médias seguidas das mesmas letras nas colunas não diferem entre si pelo teste de Tukey a $5 \%$.

Tabela 8 - Efeitos do ácido aconítico (AA) sobre a germinação e o crescimento do caule e da raiz em guanxuma, em diferentes locais do Estado do Paraná, em 2007

\begin{tabular}{|c|c|c|c|c|c|c|}
\hline \multirow{2}{*}{ Locais (11) } & \multicolumn{2}{|c|}{ Germinação (\%) } & \multicolumn{2}{|c|}{ Caule $(\mathrm{cm})$} & \multicolumn{2}{|c|}{ Raiz (cm) } \\
\hline & Sem AA & Com AA & Sem AA & Com AA & Sem AA & Com AA \\
\hline São Sebastião Amor & $61,0 \mathrm{aA}^{-1}$ & $49,0 \mathrm{aB}$ & $1,63 \mathrm{acA}$ & $0,65 \mathrm{aB}$ & $2,05 \mathrm{abA}$ & $0,15 \mathrm{aB}$ \\
\hline Jataizinho & $43,5 \mathrm{bA}$ & $36,0 \mathrm{abA}$ & $1,30 \mathrm{bdA}$ & $0,35 \mathrm{aB}$ & $1,85 \mathrm{abA}$ & $0,15 \mathrm{aB}$ \\
\hline IV Centenário & $36,5 \mathrm{bdA}$ & $25,5 \mathrm{bcB}$ & $1,68 \mathrm{acA}$ & $0,48 \mathrm{aB}$ & $0,75 \mathrm{cA}$ & $0,25 \mathrm{aA}$ \\
\hline Tupãssi & $31,0 \mathrm{bdA}$ & $24,0 \mathrm{bcA}$ & $1,63 \mathrm{acA}$ & $0,58 \mathrm{aB}$ & $1,88 \mathrm{abA}$ & $0,20 \mathrm{aB}$ \\
\hline Jesuítas & $29,5 \mathrm{bdA}$ & $21,5 \mathrm{bdA}$ & $2,08 \mathrm{abA}$ & $0,53 \mathrm{aB}$ & $2,00 \mathrm{abA}$ & $0,45 \mathrm{aB}$ \\
\hline São Pedro do Ivaí & $29,5 \mathrm{bdA}$ & $23,0 \mathrm{bdA}$ & $2,10 \mathrm{aA}$ & $0,48 \mathrm{aB}$ & $1,63 \mathrm{bcA}$ & $0,20 \mathrm{aB}$ \\
\hline Cafelândia & $26,5 \mathrm{cdA}$ & $25,5 \mathrm{bcA}$ & $2,33 \mathrm{aA}$ & $0,43 \mathrm{aB}$ & $2,48 \mathrm{abA}$ & $0,28 \mathrm{aB}$ \\
\hline Santa Terezinha Itaipu & $25,5 \mathrm{cdA}$ & $25,0 \mathrm{bcA}$ & $1,73 \mathrm{acA}$ & $0,40 \mathrm{aB}$ & $2,63 \mathrm{a} A$ & $0,15 \mathrm{aB}$ \\
\hline Floresta & $18,5 \mathrm{deA}$ & $26,5 \mathrm{bcA}$ & $2,18 \mathrm{aA}$ & $0,45 \mathrm{aB}$ & $1,75 \mathrm{abA}$ & $0,10 \mathrm{aB}$ \\
\hline Céu Azul & $15,0 \mathrm{de} A$ & $16,5 \mathrm{cdA}$ & $1,28 \mathrm{cdA}$ & $0,78 \mathrm{aB}$ & $1,65 \mathrm{acA}$ & $0,55 \mathrm{aB}$ \\
\hline São Miguel do Iguaçu & $4,0 \mathrm{eA}$ & $7,5 \mathrm{dA}$ & $0,58 \mathrm{dA}$ & $0,18 \mathrm{aA}$ & $0,75 \mathrm{cA}$ & $0,30 \mathrm{aA}$ \\
\hline Médias & $29,1 \mathrm{~A}$ & $25,5 \mathrm{~B}$ & $1,7 \mathrm{~A}$ & $0,5 \mathrm{~B}$ & $1,8 \mathrm{~A}$ & $0,3 \mathrm{~B}$ \\
\hline $\mathrm{CV} \%$ & \multicolumn{2}{|c|}{26,6} & \multicolumn{2}{|c|}{34,4} & \multicolumn{2}{|c|}{49,1} \\
\hline
\end{tabular}

1/ Médias seguidas pelas mesmas letras, minúsculas nas colunas e maiúsculas nas linhas, não diferem entre si pelo teste de Tukey a 5\%. 
Ácido aconítico em sementes de espécies de plantas ...

Tabela 9 - Efeitos do ácido aconítico (AA) sobre o desenvolvimento de fungos endofíticos em sementes de guanxuma, em diferentes locais do Estado do Paraná, em 2007

\begin{tabular}{|c|c|c|c|c|c|c|}
\hline \multirow{2}{*}{ Locais (11) } & \multicolumn{2}{|c|}{ Fusarium (\%) } & \multicolumn{2}{|c|}{ Aspergillus (\%) } & \multicolumn{2}{|c|}{ spp. (\%) } \\
\hline & Sem AA & Com AA & Sem AA & Com AA & Sem AA & Com AA \\
\hline São Sebastião Amor & $17,3 \mathrm{bdB}^{1 /}$ & $36,5 \mathrm{abA}$ & $0,0^{\mathrm{NS}}$ & 0,5 & $22,0 \mathrm{ceB}$ & $44,0 \mathrm{abA}$ \\
\hline Jataizinho & $19,0 \mathrm{bcB}$ & $30,0 \mathrm{abA}$ & 1,0 & 5,0 & $29,0 \mathrm{bdB}$ & $46,5 \mathrm{aA}$ \\
\hline IV Centenário & $3,5 \mathrm{bdA}$ & $3,0 \mathrm{eA}$ & 4,5 & 12,5 & $17,0 \mathrm{deA}$ & $25,0 \mathrm{ceA}$ \\
\hline Tupãssi & $1,0 \mathrm{dA}$ & $5,5 \mathrm{deA}$ & 3,0 & 2,5 & $9,5 \mathrm{eA}$ & $11,0 \mathrm{eA}$ \\
\hline Jesuítas & $11,5 \mathrm{bdA}$ & $10,5 \mathrm{ceA}$ & 1,0 & 2,5 & $21,5 \mathrm{ceA}$ & $18,5 \mathrm{deA}$ \\
\hline São Pedro do Ivaí & $40,0 \mathrm{aA}$ & $26,0 \mathrm{acB}$ & 2,0 & 0,5 & $46,5 \mathrm{aA}$ & $34,0 \mathrm{adB}$ \\
\hline Cafelândia & $20,5 \mathrm{bA}$ & $21,5 \mathrm{adA}$ & 5,5 & 11,0 & $36,0 \mathrm{acA}$ & $41,0 \mathrm{acA}$ \\
\hline Santa Terezinha Itaipu & $13,0 \mathrm{bdA}$ & $20,0 \mathrm{beA}$ & 0,0 & 0,5 & $17,0 \mathrm{deA}$ & $22,5 \mathrm{deA}$ \\
\hline Floresta & $4,0 \mathrm{bdA}$ & $7,0 \mathrm{deA}$ & 2,0 & 16,5 & $18,5 \mathrm{deA}$ & $28,0 \mathrm{bdA}$ \\
\hline Céu Azul & $39,5 \mathrm{aA}$ & $37,5 \mathrm{aA}$ & 1,5 & 11,0 & $41,5 \mathrm{abA}$ & $48,5 \mathrm{aA}$ \\
\hline São Miguel Iguaçu & $2,0 \mathrm{cdB}$ & $10,5 \mathrm{ceA}$ & 0,0 & 0,0 & $11,5 \mathrm{eB}$ & $18,0 \mathrm{deA}$ \\
\hline Médias & $15,6 \mathrm{~A}$ & $18,9 \mathrm{~A}$ & $1,9 \mathrm{~A}$ & $5,7 \mathrm{~A}$ & $24,6 \mathrm{~B}$ & $30,6 \mathrm{~A}$ \\
\hline $\mathrm{CV} \%$ & \multicolumn{2}{|c|}{50,0} & \multicolumn{2}{|c|}{91,8} & \multicolumn{2}{|c|}{27,7} \\
\hline
\end{tabular}

1/ Médias seguidas pelas mesmas letras, minúsculas nas colunas e maiúsculas nas linhas, não diferem entre si pelo teste de Tukey a 5\%.

médios do caule e da raiz foram reduzidos em 70,6 e $83,3 \%$, respectivamente.

As espécies de fungos endofiticos predominantes nas sementes de guanxuma foram Fusarium e Aspergillus, entre outras, como Bipolaris, Rhizopus e Penicillium, incluídas no somatório das espécies (Tabela 9). A aplicação de AA aumentou a infestação de Fusarium em três locais e, igualmente, no somatório das espécies observadas, sendo significativo o aumento de infestação obtido para as médias. No somatório de fungos, o aumento médio da infestação provocada pelo AA foi de 24,6 a $30,6 \%$.

\section{CONSIDERAÇÕES GERAIS}

A germinação das sementes coletadas das espécies de amendoim-bravo, picão-preto, corda-de-viola e guanxuma mostrou variações das espécies entre os locais amostrados. O ácido aconítico afetou a germinação das sementes, o crescimento do caule e, de modo mais significativo, o crescimento das raizes das espécies, excetuando-se a germinação de corda-de viola. Amendoim-bravo apresentou alternância de efeitos inibitórios do AA, numa ampla escala de germinação. Aplicações de AA estimularam o crescimento de fungos endofíticos na maioria dos locais de coleta das sementes, variáveis com a planta daninha, com predominância de Fusarium e Aspergillus.
A escarificação das sementes de corda-deviola resultou em aumentos da germinação, sendo ao mesmo tempo muito significativos os aumentos da infestação de fungos endofiticos em alguns locais. A presença de fungos nas sementes não germinadas é manifestada após a apresentação dos efeitos alelopáticos, dias mais tarde.

Entre os fatores relacionados ao grau de germinação das diferentes espécies de plantas daninhas estão as variações na produção de "sementes duras", influenciadas pelo ano de produção das sementes (Stoller \& Wax, 1973), incluindo as condições de temperatura e luz a que a planta-mãe foi submetida no período de formação (Probert et al., 1985). A germinação das espécies está associada a fatores genéticos e ambientais, que determinam o seu grau de dormência (Chancellor, 1982).

Os efeitos do AA sobre as espécies de plantas daninhas deverá reduzir o banco de sementes no solo e a sua germinação, resultando em menor emergência e competição com uma cultura econômica. Além disso, com a redução de altura, ocorrerão restrições à captação de luz. Com a redução do comprimento da raiz - o efeito mais importante haverá restrições à competição com a cultura por água e nutrientes. Em períodos de seca, o estabelecimento de espécies daninhas e sua competição com a cultura seriam desfavorecidos. Finalmente, os efeitos do ácido aconítico podem traduzir-se na redução do período de sobrevivência dos bancos de sementes. 


\section{AGRADECIMENTOS}

Aos que contribuíram com esta publicação e, de modo especial, ao apoio financeiro dado pelo CNPq.

\section{LITERATURA CITADA}

ADEGAS, F. S.; VOLL, E.; PRETE, C. E. C. Embebição e germinação de sementes de picão-preto (Bidens pilosa).

Planta Daninha, v. 21, n. 1, p. 21-25, 2003.

AZEVEDO, J. L.; MELO, I. S. (Eds.). Ecologia microbiana Jaguariúna: Embrapa Meio Ambiente-CNPMA, 1998. 488 p.

CHANCELLOR, R. J. Weed seed investigations. In: THOMPSON, J. R. Advances in research and technology of seeds. Wageningen: International Seed Testing Association, 1982. p. 9-29. Part 7

CHANDLER, J. M.; MUNSON, R. L.; VAUGHAN, C. E. Purple moonflower emergence, growth, reproduction. Weed Sci., v. 25, p. 163-7, 1977.

FRIEBE, A. et al. Phytotoxins from shoot extracts and root exudates of Agropyron repens seedlings. Phytochemistry, v. 38, n. 5, p. 1157-1159, 1995.

GOODWIN, T. W.; MERCER, E. I. Introduction to plant biochemistry. New York: Pergamon Press, 1983. 677 p.

HARPER, J. L.; BENTON, R. A. The germination of seeds on the surface of a water supplying substrate. J. Ecol., v. 54, n. 1, p. 151-166, 1966.

HANINE, H.; MOURGUES, J.; MOLINIER, J. Aconitic acid removal during cane juice clarification. Inter. Sugar J., v. 92, n. 1103 , p. $219-220,1990$

HEES, PAW-VAN et al. Low molecular weight organic acids and their Al-complexes in soil solution - composition, distribution and seasonal variation in three podzolized soils. Special issue: The podzolization process. Geoderma, v. 94 , n. $2-3-4$, p. $173-200,2000$

JOHNSTON, T. J.; MURRAY, D. S.; WILLIAMS, J. C Germination and emergence of baloonvine (Cardiospermum halicacabum) compared with soybean seeds. Weed Sci., v. 27, p. $73-76,1979$.

MIKUSINSKI, O. M. Teste de embebição e germinação em sementes de Ipomoea aristolochiaefolia. R. Bras. Sementes, v. 9 , n. 3 , p. 103-108, 1987
PRETE, C. E. C.; NUNES JR., J.; MENTEN, J. O. M. Fungos associados a sementes de plantas daninhas. Summa Phytopathol., v. 10, n. 2, p. 260-267, 1984.

PROBERT, R. J.; SMITHM, R. D.; BIRCH, P. Germination responses to light and alternating temperatures in European populations of Dactylis glomerata L. I. Variability in relation to origin. New Phytol., v. 99, p. 305-316, 1985.

REDDY, K. N.; SINGH, M. Germination and emergence of hairy beggarticks (Bidens pilosa). Weed Sci., v. 40, n. 2, p. 195-199, 1992.

RUSTAMANI, M. A. et al. Further observations on the relationship between aconitic acid contents and aphid densities on some cereal plants. B. Res. Inst. Biores., v. 1, n. 1, p. 9-20, 1992.

STOLLER, G. W.; WAX, L. M. Periodicity of germination and emergence of some annual weeds. Weed Sci., v. 21, n. 6 , p. $574-580,1973$

THOMPSON, F.; SCHAEFER, S. C.; MADISON, J. T. Role of aconitate isomerase in trans-aconitate accumulation in plants. J. Agric. Food Chem., v. 45, n. 9, p. 3684-3688, 1997.

VOLL, E.; KARAM, D.; GAZZIERO, D. L. P. Dinâmica de populações de trapoeraba (Commelina benghalensis L.) sob manejos de solo e de herbicidas. Pesq. Agropec. Bras., v. 32, n. 6, p. 571-578, 1997.

VOLL, E. et al. Dinâmica do banco de sementes de plantas daninhas sob diferentes sistemas de manejo do solo.

Planta Daninha, v. 19, n. 2, p. 171-178, 2001.

VOLL, E. et al. Chemical interactions of Brachiaria plantaginea with Commelina benghalensis and Acanthospermum hispidum in soybean cropping systems. J. Chem. Ecol., v. 30, n. 7, p. 1467-1475, 2004

VOLL, E.; VOLL, C. E.; VICTÓRIA FILHO, R. Allelopathic effects of aconitic acid on wild poinsettia (Euphorbia heterophylla) and morningglory (Ipomoea grandifolia). J. Environ. Sci. Health, v. 40, n. 1, p. 69-75, 2005.

WATANABE, $\mathrm{K}$. et al. Detection and molecular analysis of plant- and insect-associated bacteria harboring aconitate isomerase involved in biosynthesis of trans-aconitic acid as antifeedant in brown planthoppers. Cur. Microbiol., v. 35, n. 1, p. $97-102,1997$ 